\title{
Comparative Evaluation of Neonates Delivered by Elective Caesarean Section under General Anaesthesia and Spinal Anaesthesia with Intrathecal Opioid (Fentanyl)
}

\author{
Dr Gaurav Chopra DA MD, Sarvesh Kumar MD
}

\begin{abstract}
Background: To compare general and spinal anaesthesia with respect to neonatal outcome and observation of post operative adverse effects born by elective caesarean section.

Methods: 60 female parturients women were posted for elective caesarean section (CS) at appropriate gestational age under spinal anaesthesia (SA) and general anaesthesia (GA). There were equal no. of patients $(n=30)$ for both the groups, GA and SA.

Maternal age, weight, height, ASA grading, neonatal Apgar score (1 and 5 minute) were recorded.[1] Early neonatal neurobehavioural assessment using Scanlon's ENNS (Early Neonatal Neurobehavioural Scale) was done 8-10 hours post birth at the nursery. [2]

Post operative adverse effects-Nausea, Vomiting, Pruritis, Shivering, Sedation and Post dural puncture headache (PDPH) were documented.

Statistical analysis performed using student 't' test and coefficient of variance.

Results: Maternal age, weight, height and ASA grading were not different between the study groups. Apgar scores were overall better in SA group at 1 minute and similar at 5 minute between both the groups. But no statistical significance seen between the groups.

Early neurobehavioural assessment was found to be different with regard to Moro's, Sucking and Startle reflexes.

Post operative adverse effects were more in GA group except pruritis and PDPH seen only in SA group.

Conclusion: Spinal anaesthesia with intrathecal opioid (Fentanyl) is the preferred method of administering anaesthesia in elective caesarean section for better neonatal outcome.

Keywords: Anaesthesia-General-Spinal-Neonate-Apgar-Caesarean-Neurobehavioural
\end{abstract}

\section{Introduction}

The selection of an anaesthetic technique for Caesarean delivery depends on several surgical, anaesthetic \& maternal factors. Surgical factors include the indication for CS and also the degree of urgency for delivery. Local anaesthetics (LA) alone were used for many years in SA, of which bupivacaine is the most frequently used LA. Opioids have been a choice in SA to improve the antinociceptive effect of local anaesthetics (LA), of which Fentanyl is commonly used intrathecally in Caesarean sections. [3,4]

Opioids are synergistic with LAs and intensify sensory blockade without increasing motor blockade, there is prolonged duration of effective analgesia and improved intra-operative analgesia, reduced LA dose requirements, reduced intra-operative antiemetic medication, $[11,12]$

Apgar score is so designed to clearly identify depressed infants requiring resuscitation and thereafter to follow their progress over first few minutes of life ( 1 and 5 minutes post-birth). (2)

Other advantages of this score are: good co-relation with acid-base status, good prognosis of neonatal morbidity and mortality.

Neonatal neurobehavioural tests are instrumental in such conditions, of which an important and widely used is the Scanlon's Early Neonatal Neurobehavioural Scale (ENNS), which is based on Prechtl and Beintema Neurological Examination and the BrazeltonBehavioural Scale.

These tests are used for studying the effects on the neonate, of maternal medication and anaesthetic techniques. This study primarily aims at comparing the effects of GA and SA with opioid (Fentanyl) on the neonate, utilizing Apgar score and Neurobehavioural assessment in planned Caesarean sections.

\section{Material And Methods}

This study was carried out over a period of twelve months. After obtaining hospital ethics committee approval and fully informed consent, 60 healthy parturient women with singleton uncomplicated pregnancy appropriate for gestational age were selected for the study.

The patients were selected from a cohort classified as American Society of Anaesthesiologists (ASA) grade I or II. 
All mothers were posted for elective CS .Randomization depended on as per mother's choice of anaesthesia (GA or SA) and standard contraindications to GA and SA were taken into consideration.

The patients were divided accordingly into following 2 groups:

Group A: Neonates delivered by Caesarean Section under General Anaesthesia.

Group B: Neonates delivered by Caesarean Section under spinal Anaesthesia with intrathecal fentanyl.

Exclusion criteria:

Complicated pregnancies (e.g. Placenta praevia, abruptio placentae etc.).

Multiple pregnancies.

Severe pregnancy induced hypertension (P.I.H).

Morbidly obese patients (BMI >40).

Acute fetal distress.

Pregnancies with bleeding disorders.

A day prior to surgery all patients posted for surgery underwent pre-anaesthetic check up and routine investigations.

Consent and fasting status of the patients was confirmed on arrival in the pre induction room. Intravenous line was secured with $18 \mathrm{G}$ cannula.

The infusion was started with intravenous fluid. Foley's catheterization was done after shifting the patient on the operation table after explaining the procedure.

ECG leads were attached and heart rate (HR), systolic blood pressure (SBP), diastolic blood pressure (DBP), mean arterial pressure (MAP), $\mathrm{SpO}_{2}$ and ECG monitoring was done.

General Anaesthesia

The patients were placed in the supine position with head on a standard pillow of $7 \mathrm{~cm}$ height.

Preoxygenation done with 100\% oxygen by face mask delivered through Bain circuit for 3 minutes.

Anaesthesia was induced with sleep dose of injection thiopentone (4-7 mg/kg i.v.) sufficient to obtund the eyelash reflex, followed by intravenous injection of succinyl choline $1-2 \mathrm{mg} / \mathrm{kg}$ and manual IPPV (Intermittent Positive Pressure Ventilation) followed by orotracheal intubation with the proper size, low pressure high volume polyvinyl chloride endotracheal tube.

Maintenance of anaesthesia was done with $66 \%$ nitrous oxide in 33\% oxygen and $0.5-1 \%$ isoflurane, muscle relaxant injection vecuronium bromide $0.1 \mathrm{mg} / \mathrm{kg}$ i.v, gentle manual intermittent positive pressure ventilation was started via endotracheal tube through Bains circuit.

After delivery of baby injection oxytocin $20 \mathrm{U}$ given i.v.slowly after clamping of cord. Injection fentanyl 2 $\mathrm{mcg} / \mathrm{kg}$ body weight was given i.v.

After completion of surgery neuromuscular blockade was reversed. Patients were shifted to post anaesthesia recovery area.

Spinal Anaesthesia:

All mothers were given preloading with normal saline (NS) or Ringer Lactate (RL) at rate of $15-20 \mathrm{ml} / \mathrm{kg}$ about 15-20 minutes earlier. Under all aseptic precautions, with the parturient in left lateral position the back of the patient was cleaned and draped. After preparing the back with antiseptic solution, local block with $2 \%$ lignocaine was given

A 23 G Quincke's needle was inserted into the subarachnoid space, preferably into the L3-4 interspace by the midline approach with the aperture of the needle directed laterally.

Once free flow of CSF is seen, the local anaesthetic (LA) solution of $2.25 \mathrm{ml}(11.25 \mathrm{mg})$ of $0.5 \%$ hyperbaric bupivacaine mixed with 15 micrograms $(\mu \mathrm{g})$ of fentanyl was injected intrathecally. [5,6]

Immediately after the block each parturient was placed supine with $15-20$ degrees towards left uterine displacement, using a right sided wedge. Oxygen at 4-6 litres per minute was given via face mask.

Heart rate, $\mathrm{SpO} 2$, non-invasive arterial blood pressure were recorded before the block and at every 3 minutes for first 15 minutes and then every 5 minutes subsequently, until the end of surgery.Sensory and motor blocks were assessed.

Intraoperative changes in maternal haemodynamics were noted.

Surgical Technique:

It was uniform in all patients and included exteriorization of the uterus with placing of double clamps on the umbilical cord of all babies immediately after birth.

Neonatal Resuscitation and Assessment:

Resuscitation of babies was done as per the protocol prescribed by the neonatal Advanced Life Support advocated by the American Paediatric Association.

At delivery, for evaluation of neonate, Apgar scores were assigned at one and five minutes by a paediatrician. 
It was based on the appearance (colour), pulse rate, grimace (reflexes), muscle tone (activity) and respiratory effort of neonate each carrying a score from 0 to 2 . Total scores indicated are $8-10=$ good; $3-7=$ fair; $0-2=$ poor condition.[7,8]

The neonate was also evaluated with a modification of Scalon's Early Neonatal Neurobehavioural Scale (ENNS) by a paediatrician who was not aware regarding the anaesthesia technique (GA or SA) administered to the mother. This is as practiced at Neonatal Intensive Care Unit (NICU) at ourHospital, at 8-12 hours post birth. Scores were assigned from 0-3 to each of six neonatal reflexes observed namely Moro`s, Rooting, Sucking, Startle, Grasp and Tonic neck reflex.[9,10]

On the first post operative day, the parturients were interviewed for post operative adverse effects nausea, vomiting, pruritus, shivering, sedation and post dural puncture headache (PDPH) and documentation was done accordingly.

\section{Data Analysis:}

Student' $t$ ' test was used to calculate significance using ' $p$ ' value.

Comparison between the two groups was done using coefficient of variance.

III. Results

Table 1: - Neonatal Evaluation Apgar Scores

\begin{tabular}{|c|c|c|}
\hline & $\begin{array}{c}\text { Group A (GA) } \\
(\mathbf{n}=\mathbf{3 0}) \\
\text { Mean } \pm \text { SD }\end{array}$ & $\begin{array}{c}\text { Group B (SA) } \\
(\mathbf{n}=\mathbf{3 0}) \\
\text { Mean } \pm \text { SD }\end{array}$ \\
\hline $1 \mathrm{~min}$ & $7.56 \pm 1.20$ & $7.96 \pm 0.60$ \\
\hline $5 \mathrm{~min}$ & $8.76 \pm 0.84$ & $8.96 \pm 0.48$ \\
\hline
\end{tabular}

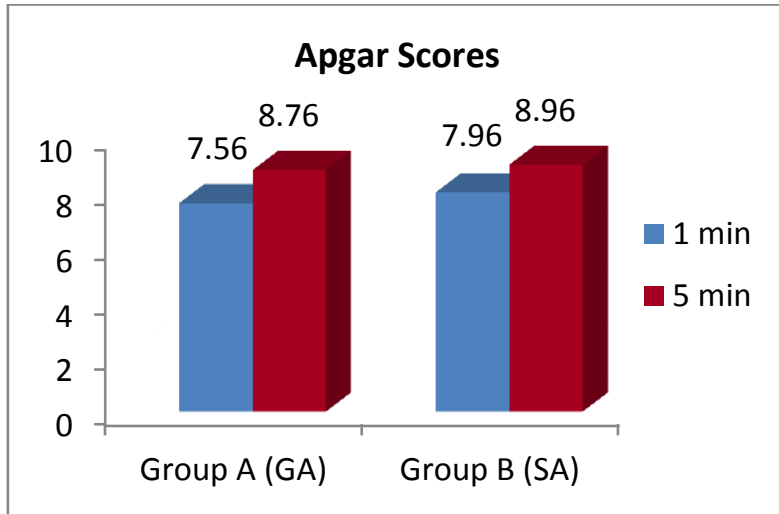

Table 2: - Neonatal Evaluation Neurobehavioural Assessment (ENNS Scale)

\begin{tabular}{|c|c|c|}
\hline & $\begin{array}{c}\text { Group (GA) } \\
\text { Mean } \pm \text { SD }(\mathbf{n}=30)\end{array}$ & $\begin{array}{c}\text { Group (B) } \\
\text { Mean } \pm \text { SD (n=30) }\end{array}$ \\
\hline Moro's & $2.36 \pm 0.54$ & $2.83 \pm 0.37$ \\
\hline Sucking & $2.40 \pm 0.55$ & $2.70 \pm 0.45$ \\
\hline Rooting & $2.46 \pm 0.45$ & $2.60 \pm 0.48$ \\
\hline Startle & $2.16 \pm 0.45$ & $2.50 \pm 0.50$ \\
\hline Grasp & $2.13 \pm 0.61$ & $2.40 \pm 0.61$ \\
\hline Tonic neck reflex & $1.93 \pm 0.72$ & $2.13 \pm 0.42$ \\
\hline
\end{tabular}

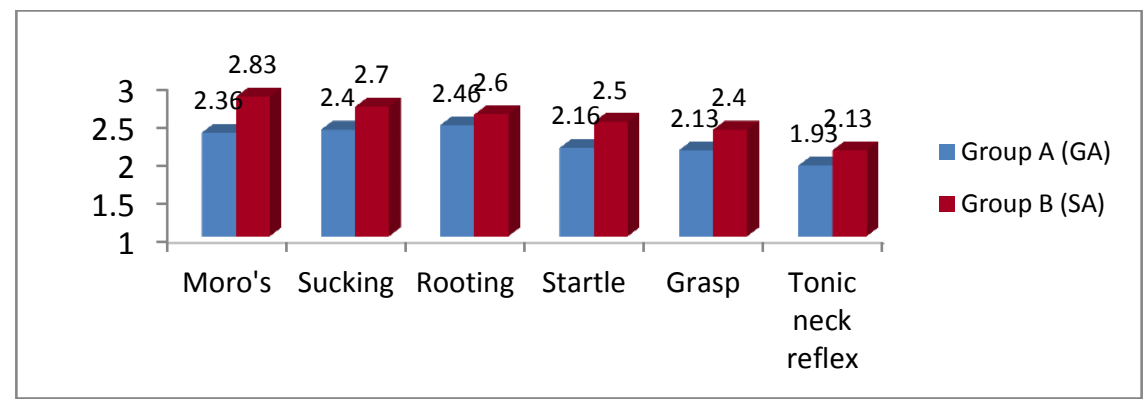


Table 3: - Incidence of Post Operative adverse effects

\begin{tabular}{|c|c|c|}
\hline & $\begin{array}{c}\text { Group A (GA) } \\
(\mathrm{n}=30) \\
\text { Number }(\%)\end{array}$ & $\begin{array}{c}\text { Group B (SA) } \\
(\mathrm{n}=30) \\
\text { Number }(\%)\end{array}$ \\
\hline Nausea & $8(27)$ & $6(20)$ \\
\hline Vomiting & $3(17)$ & $2(13)$ \\
\hline Pruritis & NA & $4(13)$ \\
\hline Shivering & $6(20)$ & $4(13)$ \\
\hline Sedation & $7(23)$ & $1(3)$ \\
\hline PDPH & NA & $1(3)$ \\
\hline
\end{tabular}

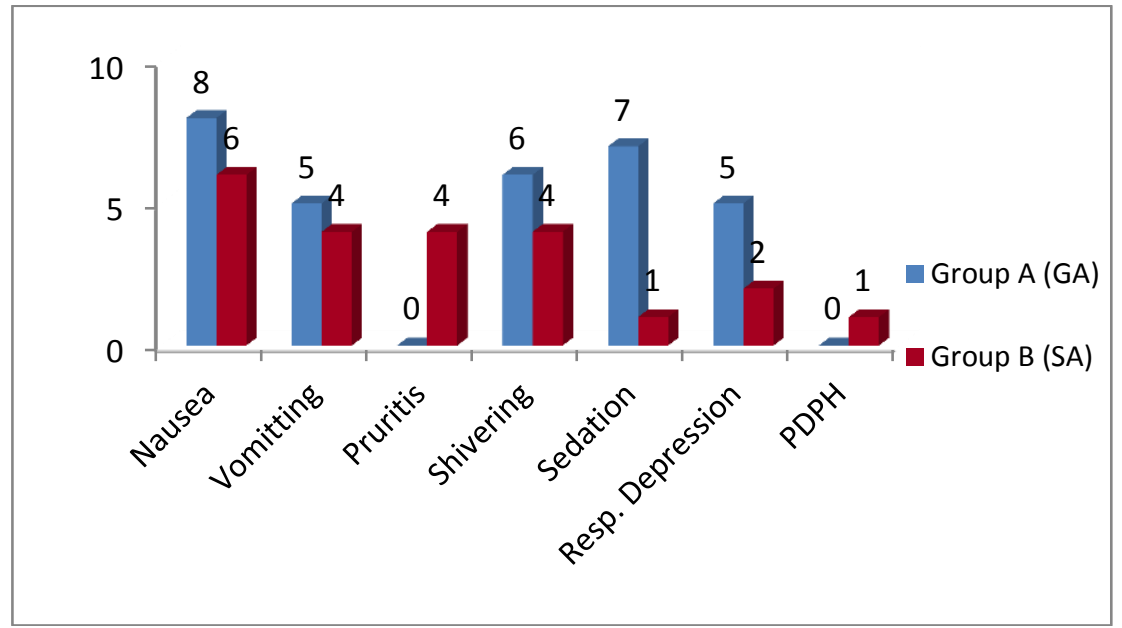

\section{Discussion}

SA among the neuraxial blocks in obstetric patients needs more strict dose calculations as the drugs are directly injected in IT space which is reduced in pregnancy. With minimum dose changes, the chances of complications and side effects are enhanced.

\section{Neonatal Evaluation}

\section{Apgar Scores}

The mean of Apgar scores at 1 minute was $7.56 \pm 1.20$ in group A (GA) and $7.96 \pm 0.60$ in group B (SA).

The mean of Apgar scores at 5 minutes was $8.76 \pm 0.84$ in group A (GA) and $8.96 \pm 0.48$ in group B (SA).

There was statistically no significant differences between the groups at 1 and 5 minute $(p>0.05)$.

Neonates born to mothers in group B (SA) had better outcomes than those born to mothers in group A (GA). In five cases of group A (GA) higher incidence of low Apgar score at 1 minute was observed, while no difference in Apgar score was observed at 5 minute.

\section{Neurobehavioural Assessment (ENNS Scale)}

Neonates born under GA and SA were compared using 6 reflexes as practiced at NICU at our Hospital, at 8-12 hours post birth.
a) Moro's
b) Sucking
c) Rooting
d) Startle
e) Grasp
f) Tonic neck reflex.

The mean of Moro's reflex was $2.36 \pm 0.54$ in group A (GA) and $2.83 \pm$ group B.

The mean of Grasp reflex was $2.13 \pm 0.61$ in group A and $2.40 \pm 0.61$ in group B.

The mean of Tonic neck reflex was $1.93 \pm 0.72$ in group A and $2.13 \pm 0.42$ in group B.

Moro's, Sucking and Startle reflexes were found to be statistically significant between the groups A and B $(\mathrm{p}<0.05)$.

On further statistical analysis it was found that Moro's, Sucking and Startle reflexes were better in group B (SA); though all reflexes studied were found to be more vigorous in group B (SA).[13,14]

The depression of 6 neonatal reflexes namely Moro`s, Sucking, Rooting, Startle, Grasp and Tonic neck reflex studied was primarily related to the effect of intravenously administered anaesthetic drugs to the mothers namely thiopentone sodium, fentanyl citrate and vecuronium bromide. Inhalation agent isoflurane was also used in low concentration $(0.5-1 \%)$.

Out of these, thiopentone appears to be the main culprit due to its slow metabolism and presence of intermediate 
metabolites. These can cross the uteroplacental barrier in significant amount and cause neonatal depression. The metabolites of thiopentone specially pentobarbital depresses Moro`s and Sucking reflexes to a greater degree. They also have an effect on the EEG (electroencephalogram) of the neonate for several days.

The highly ionized property of muscle relaxants impedes placental transfer resulting in minimal effects on fetus. Although most opiates readily cross the placenta, their effect on neonates at delivery vary considerably. Fentanyl does not appear to cause depressant effect on neonates and in our study it was administered after delivery of baby, so it is not implicated (30).

All inhalational agents freely cross the placenta and produce insignificant fetal depression when given in limited doses $(\leq 1 \mathrm{MAC})$.

Thus in our study, it appears finally that thiopentone is the main responsible agent for the poor neonatal outcome in patients delivered under GA.

\section{Incidence of Post Operative Adverse Effects}

1) The incidence of nausea was $27 \%$ of patients in group I and $20 \%$ in group II. No statistically significant difference was seen between the groups.

The incidence of vomiting was $10 \%$ of patients in group I and $7 \%$ in group II. No statistically significant difference was seen between the groups.

Postoperative nausea and vomiting (PONV) may be due to intravenously administered opioids, use of nitrous oxide, female sex and increased duration of surgery in cases of GA.[15,16]

These may also indicate subclinical visceral pain due to uterine manipulation and peritoneal handling intraoperatively.

Hypotension defined as $\geq 25 \%$ decreases in systolic arterial pressure from preanaesthetic

values, is an important cause of vomiting. (7).

2) Pruritis was observed only in 4 patients of group B (SA with intrathecal fentanyl), the incidence being $7 \%$.

Pruritis induced by neuraxial opioids is likely due to activation of $\mu$-opioid receptors located both supraspinally and in the dorsal horn (substantiagelatinosa) of the spinal leading to truncalpruritis and further cephalad migration of the opioid in CSF and subsequent interaction with opioid receptors in the trigeminal nucleus leading to facial pruritis.

None of the patients in our study groups required antipruritic treatment as it was of short duration, low to mild intensity and involving only the tip of nose and facial region.

3) Shivering was observed in $20 \%$ of patients in group A (GA) and $13 \%$ in group B (SA). No statistical difference was seen between the groups.

Probable causes of shivering are relaxed to decrease of core temperature such as:-

1. Sympathetic blockage resulting in peripheral vasodilatation, increased cutaneous blood flow and heat loss is the skin.

2. Cold operating room or rapid infusion of crystalloid solution at room temperature.

3. Direct effects of cold anaesthetic solution upon thermosensitive structures within the spinal cord.

4. Use of intravenous inducing agent thiopentone and inhalational agent isoflurane in patients undergoing general anaesthesia as these agents cause vasodilatation.

Injection tramadol $50 \mathrm{mg}$ i.v and $50 \mathrm{mg}$ in drip was administered to patients of both groups to control shivering in our study.

Shivering associated with SA in patients undergoing CS is one of the common problems. It is very uncomfortable for the patients and may interfere with the monitoring. It also increases oxygen consumption, lactic acidosis and carbon dioxide production. $[17,18]$

4) Sedation was observed in $23 \%$ of patients in group A and $3 \%$ in group B. Statistically significant difference was seen between the groups.

Sedation in group $\mathrm{A}(\mathrm{GA})$ appeared postoperatively due to effect of intravenous anaesthetic drugs mainly thiopentone, opioids like fentanyl, inhalation agent isoflurane are implicated and the effect appears to be dose related.

Sedation in group B (SA) postoperatively was due to effect of benzodiazepine midazolam given i.v. $1+2 \mathrm{mg}$ intraoperatively for anxiolysis and calming the patient as the patient in our study was very anxious preoperative. $100 \%$ oxygen by simple face mask was given to all sedated patients in both the groups to avoid any fall in oxygen saturation due to frequently associated depression of ventilation.

5) PDPH (post dural puncture headache) was present in only 1 patient (3\%) of patients of group B.

PDPH occurred in patient of our study due to noncompliance of postoperative orders and early ambulation before 24 hours.

PDPH as seen in patient in our study was bilateral, frontal and throbbing in type. It was aggravated by sitting or standing and relieved by lying down flat. It results from leakage of CSF from a dural defect and decreased 
intracranial pressure.

Conservative treatment involves recumbent positioning, intravenous fluid administration, caffeine in the form of tea and coffee, plenty of oral fluids.[19,20]

\section{Conclusions}

Following conclusions were drawn:

Neonatal outcome in terms of Apgar score was found to be better in group B (SA) as compared to group A (GA).Statistically no significant difference was observed between the groups.

Early neonatal neurobehavioural assessment using ENNS scale was better in group B (SA) as compared to group A (GA).

The reflexes were overall more vigorous in group B than group A (GA). Moro's, Sucking and Startle were found to be statistically significant between the groups. On further analysis, it was found that Moro's, Sucking and Startle reflexes were much better in group B (SA) than group A (GA).

Postoperative nausea and vomiting was observed to be more in group A (GA) as compared to group B (SA)and statistically not significant between the groups.

Postoperative pruritis was observed only in group B (SA) and absent in group A (GA) and was statistically significant between the groups.

Postoperative shivering was observed more in group A (GA) in comparison to group B (SA) and was statistically not significant between the groups.

Postoperative sedation was observed more in group A (GA) as compared to group $\mathrm{B}(\mathrm{SA})$ and was statistically significant between the groups.

Postdural puncture headache was observed only in group B (SA) and absent in group A (GA) and was statistically not significant between the groups.

Thus we concluded that neonates born under GA were found to be more depressed as reflected by lower Apgar scores at 1 and 5 minute.

8-10 hours post birth and were found to be more vigorous in group B (SA) as compared to group A. Moro's, Sucking and Startle were statistically significant between the groups.

Moro's and Sucking reflexes reflect the integrity of neonatal central nervous system and are sure indicators of neonatal well being.

The lower Apgar scores and depressed ENNS assessment as reflected in group A(GA) were mainly due to effects of intravenously administered anaesthetic inducing agent thiopentone.

Post-operative adverse effects were observed to be more in group A (GA) as compared to group B (SA) .

\section{References}

[1]. Goffman D. The Effect of Anaesthesia on Apgar Score.http:// www.medscape.com/viewarticle/520775.

[2]. Cohen SE. Evaluation of the neonate. In: Shnider SM, Levinson G, editors. Anaesthesia for Obstetrics: 1st ed. Baltimore: Williams and Wilkins; 1981. p. 370-84.

[3]. Dresner MR: Anaesthesia for caesarean section. Best Pract Res ClinObstetGynaecol. 2001; 15(1): 127-43.

[4]. Ngiam SK: The addition of intrathecalsufentanil and fentanyl to bupivacaine for caesarean section. Singapore Med J. 1998; 39(7): 290-4.

[5]. Gogarten W: Spinal anaesthesia for obstetrics. Best Pract Res Clin Anaesthesiol.2003;17(3):377-92

[6]. Ong BY, Cohen MM, Palahniuk RJ: Anesthesia for Caesarean Section-Effects on Neonates .Anesth Analg.1989;68:270-5.

[7]. Kavak ZN, Basgul A, Ceyhan N: Short-term outcome of newborn infants: spinal versus general anaesthesia for elective caesarean section. A prospective randomized study. Eur J ObstetGynecolReprod Biol.2001;100(1):50-4.

[8]. Gori F, PasqualucciA,Corradetti F, MilliM,PedutoVA:Maternal and neonatal outcome after cesarean section:the impact of anesthesia.JMatern Fetal Neonatal Med.2007;20(1):53-7.

[9]. KolatatT,SomboonnanondaA,LertakyamaneeJ,ChinacotT,TritrakarnT,Muangkasem J:Effects of General and Regional Anesthesia on the neonate (a Prospective, Randomized Trial).J Med Assoc Thai.1999;82(1):40-5.

[10]. Nippon SankaFujinkaGakkai Zasshi.1983;35(4):473-80.

[11]. Hodgkinson R, Bhatt M, Kim SS, Grewal G, Marx GF: Neonatal neurobehavioural tests following caesarean section under general and spinal anaesthesia. Am J Obstet Gynecol.1978;132(6):670-4.

[12]. Hamber EA: Intrathecal Lipophilic Opioids as Adjuncts to Surgical Spinal Anaesthesia. RegAnesth Pain Med.1999; 24(3):255-63.

[13]. Bogra J , Arora N, Srivastava P : Synergistic effect of intrathecal fentanyl and bupivacaine in spinal anaesthesia for caesarean section. BMC Anesthesiol.2005;5:5.

[14]. Davies NJH, Cashman JN, editors. Lee`s Synopsis of Anaesthesia: $1^{\text {st }}$ ed. New Delhi: Butterworth-Heinemann;2006.

[15]. Abboud TK, Nagappala S, MurakawaK,DavidS,HaroutunianS,ZakarianM,etal.Comparison of the Effects of General and Regional Anaesthesia for Caesarean Section on Neonatal Neurologic and Adaptive Capacity Scores. Anaesthesia and Analgesia.1985;64:9961000 .

[16]. Dahl JB, Jeppesen IS, Jorganson H, Welterslev J, Moiniche S. Intraoperative and Postoperative Analgesic Efficacy and Adverse Effects of Intrathecal Opioids in Patients Undergoing Caesarean Section with Spinal Anaesthesia. Anaesthesiology.1999;91(6):1919-27.

[17]. Ratcliffe FM, Evans JM. Neonatal well being after elective caesarean delivery with general, spinal and epidural anaesthesia.Eur J Anaesthesiol.1993; 10: 175-181. 
[18]. Cosmi EV, Mark GF. Acid-base status of the fetus and clinical condition of the newborn following caesarean section.Am J Obstet Gynecol.1968;102: 378-82.

[19]. Choi DH, Ahn JH, Kim MH: Bupivacaine -Sparing Effect of Fentanyl in Spinal Anaesthesia for Caesarean Delivery. RegAnesth Pain Med.2000;25:240-5.

[20]. Singh H,YangJ,ThorntonK,GieseckeAH.Intrathecal fentanyl prolongs sensory bupivacaine spinal block. Can J Anesth.1995; 42(11):987-91.

[21]. Morgan, Jr GE, Mikhail MS, Murray MJ. Clinical Anaesthesiology.4 $4^{\text {th }}$ ed. Singapore: McGraw-Hill Education; 2008. 